\title{
Optical Polarimatic Characterization of Zinc Oxide Nanorods as a Photosensitizer for Photodynamic Therapy
}

\author{
Shamaraz Firdous ${ }^{1 *}$, Amanat Ali ${ }^{1}$ and Shahzad Anwar ${ }^{1,2}$ \\ ${ }^{1}$ Biophotonics Lab, National Institute of Lasers and Optronics (NILOP), Pakistan \\ ${ }^{2}$ National Center for Nanoscience and Technology (NCNST), University of Chinese Academy of Sciences, China
}

Submission: March 15, 2018; Published: May 23, 2018

"Correspondence Address: Shamaraz Firdous, Biophotonics Lab, National Institute of Lasers and Optronics (NILOP), 45650 Islamabad, Pakistan, Email: shamaraz@gmail.com

\begin{abstract}
The role of nanoparticles in diagnostic and treatment of cancer and other malignant diseases is gaining much research attention. The zinc oxide nanorods (ZnO NRs) are one of the most studied and promising semiconductor materials for applications in materials and medical sciences. In this study, we present optical properties of $\mathrm{ZnO}$ nanorods using Mueller matrix polarimetry to be suitable for its application in photodynamic therapy (PDT). Mueller matrix spectroscopic system capable of acquiring complete polarization information from single nanoparticle to nanostructure in term of transmittance, depolarization, retardance, and polarizance.

The polarization properties measured from 400 to $800 \mathrm{~nm}$ wavelength of light described decreasing trend of transmission at a wavelength range of $400 \mathrm{~nm}$ to $530 \mathrm{~nm}$. This behavior of light describes Rayleigh scattering in the region and shows $\mathrm{ZnO}$ nanorods of relatively smaller diameter to excitation wavelength. Depolarization index decreases from 0.98 to 0.84 on $400 \mathrm{~nm}$ to $650 \mathrm{~nm}$ showing bulk scattering of light from randomly distributed $\mathrm{ZnO}$ nanorods. Slight change in retardance from $445 \mathrm{~nm}$ to $550 \mathrm{~nm}$ shows that light having low frequency and high wavelength passes readily than the high frequency light presenting that $\mathrm{ZnO}$ nanorods are strong, compact and composite material. Diattenuation magnitude increases with the increase in wavelength from $400 \mathrm{~nm}$ to $750 \mathrm{~nm}$ and changes abruptly at a wavelength range from $750 \mathrm{~nm}$ to $800 \mathrm{~nm}$. This irregular trend indicates that ZnO nanorods are birefringent and anisotropic. These measured polarized properties proved that ZnO NRs are suitable to use as photosensitizer in PDT for diagnostic and treatment of cancer and other malignant diseases.
\end{abstract}

Keywords: Optical diagnostic; Polarized light; Multiple scattering of light in turbid media; Depolarization; Photodynamic therapy (PDT); Photosensitizer; Zinc oxide nanorods.

\section{Introduction}

Nanotechnology and nanoparticles provided great advancement in photodynamic therapy of malignant diseases over the last decades [1-2]. Zinc oxide is a bio-safe and biocompatible material and an attractive candidate for biomedical applications [3-5]. ZnO nanorods (NRs) with high surface area to volume ratio and biocompatibility is a good candidate for efficient photosensitizer (PS) carrier system and at the same time providing intrinsic white light needed to achieve cancer cell necrosis. Drug delivery into the malignant cell is a basic requirement for effectiveness of photosensitizing systems for photodynamic therapy (PDT). For anticancer tumoricidal drugs, e.g., 5-aminolevulinic acid (ALA) along with zinc oxide (ZnO) nanoparticles (NPs) used as efficient intracellular photosensitizer carriers [6-8]. Recently, considerable interest has arisen in the optical properties of $\mathrm{ZnO}$ nano-material [9-10]. We discuss optical polarization properties of wave propagation through ZnO NRs as a photosensitizer in PDT [11]. This is the fact that polarization methods are giving all possible information's of the investigated medium, through light propagation. Scattering of light from turbid medium randomize the state of polarization and wave propagation in such a medium become rapidly depolarized. We have previously reported on depolarization of optical wave propagating through random media for different biomaterials [12-24].

The ZnO nanoparticles, nano flowers and nanorods are used not only also a potential photosensitizer but as shown light extraction efficiency in photonic devices [25]. Due to this, $\mathrm{ZnO}$ has a potential for a wide range of applications in UV and intrinsic white light emitting devices. The UV and green emission part of the white light of $\mathrm{ZnO}$ may be used to activate some biological process, such as the activation of photosensitizers for photodynamic therapy [26]. Recently photodynamic therapy (PDT) is most widely used for cancerous treatment due to side effects of the traditional treatment strategies. It is a non-invasive, 
promising and specially targeting tumoral tissue treatment. In current experimental study, we present optical and non-invasive properties of $\mathrm{ZnO}$ nanorods in order to use it as an efficient photosensitizer [27]. Some attractive features of nanomaterials lead to the development of new technologies medical diagnostic procedures and drug delivery system. Nanomaterials used in various biomedical strategies and generate reactive oxygen species (ROS) by interacting with the proteins and enzymes in human cells, which ultimately causes apoptosis or necrosis of malignant cell [28].

The use of $\mathrm{ZnO}$ nanoparticles (NPs) for biomedical applications, particularly PDT, relies on the fact that semiconductor nanomaterials could generate reactive oxygen species (ROS) and are promising candidates to become the new generation of photosensitizers. $\mathrm{ZnO}$ is an excellent candidate for photosensitizer due to its nontoxicity and ability of biodegrade with high thermal and chemical stabilities [29]. Optical polarization properties of the scattered light are crucial for fundamental understanding of the aforementioned effects because polarization plays an important role in the light-matter interactions effects. Moreover, the polarization information should also exploited to develop polarization-controlled novel schemes for contrast enhancement in biomedical imaging using Mueller matrix model. Mueller matrix is a $4 \times 4$ matrix representing the transfer function of any optical system in its interaction with polarized light and all the medium polarization properties are characteristically encoded in its various elements.
The recorded Mueller matrix analyzed to extract or quantify the intrinsic polarization properties of the medium. The resulting polarization properties, transmittance, depolarization, retardance, and polarizance, potentially utilized for quantitative analysis / interpretation of biomaterials [30,31].

\section{Material and Methods}

We have purchased the reagents including zinc acetate dehydrate and sodium hydroxide from Sigma-Aldrich Company (UK) and synthesized $\mathrm{ZnO}$ nanorods (ZnO-NRs) through hydrothermal technique. Precursor used for $\mathrm{ZnO}$ nanorods preparation are zinc acetate dehydrate $\mathrm{Zn}\left(\mathrm{CH}_{3} \mathrm{COO}\right) 2 \cdot 2 \mathrm{H}_{2} \mathrm{O}$ (98\%) and sodium hydroxide $\mathrm{NaOH}(98 \%)$ [32,33]. The Mueller matrix polarimetric system (AxioScan, Axometrics, Inc.USA.) consist on polarization state generator (PSG), polarization state analyzer (PSA), and spectrally resolved signal detection unit. Collimated white light from a mercury lamp used as an excitation source and is passed through the PSG unit for generating the input polarization states. The PSG unit consists of a horizontally oriented fixed linear polarizer P1 and a rotatable quarter wave plate (QWP1) mounted on a computer controlled rotational mount. The PSG-emerging light focused to the sample and the sample and scattered light collected by the PSA unit for the analysis of the polarization state of light and relayed for spectrally resolved signal detection. The PSA unit comprises of the same polarization components with a fixed linear polarizer (P2) and a computer controlled rotating quarter wave plate (QWP2) as shown in Figure 1 [34].

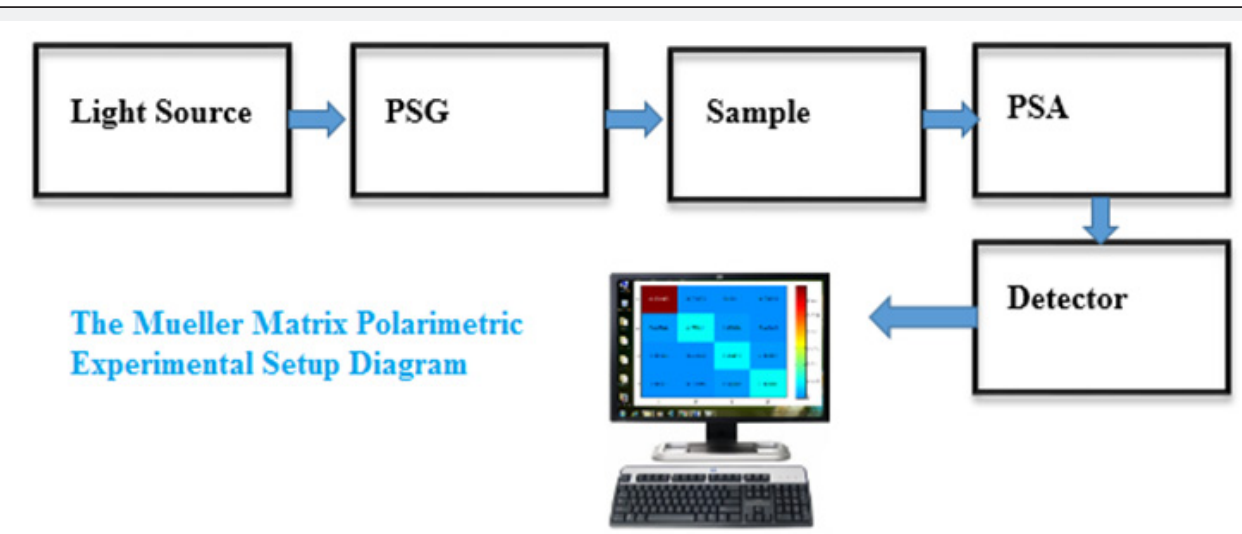

Figure 1: Experimental set up of dual rotating retarder Mueller matrix polarimeter. A sample-measuring polarimeter consists of a source, polarization state generator (PSG), the sample, a polarization state analyzer (PSA) and the detector.

\section{Result and Discussion}

In our research, we used Mueller matrix polarimetric model for the optical characterization of Zinc oxide metallic nanorods to use in photodynamic therapy (PDT). For the synthesize of photosensitizer ( $\mathrm{ZnO}$ nanoparticles), we used stock solutions of $\mathrm{Zn}\left(\mathrm{CH}_{3} \mathrm{COO}\right)_{2} \cdot 2 \mathrm{H}_{2} \mathrm{O}(0.1 \mathrm{M}) \mathrm{s}$ prepared in $50 \mathrm{ml}$ methanol under stirring. To this stock solution, $25 \mathrm{ml}$ of $\mathrm{NaOH}$ (varying from $0.2 \mathrm{M}$ to $0.5 \mathrm{M}$ ) solution prepared in methanol added under continuous stirring in order to get the $\mathrm{pH}$ value of reactants between 8 and 11. These solutions was transferred into teflon lined sealed stainless steel autoclaves and maintained at various temperature in the range of $100-200{ }^{\circ} \mathrm{C}$ for 6 and $12 \mathrm{~h}$ under autogenous pressure. It then allowed cooling naturally to room temperature. After the reaction was complete, the resulting white solid products washed with methanol, filtered and then dried in air in a laboratory oven at $60{ }^{\circ} \mathrm{C}[35]$.

The synthesized samples characterized for their structure with optical polarized light Mueller matrix system. The demonstrated novel ability to record full polarization information over a broad wavelength range and to quantify the 


\section{Cancer Therapy \& Oncology International Journal}

intrinsic polarimetry characteristics via Mueller matrix analysis should lead to a novel route towards quantitative understanding, analysis or interpretation of a number of effects and may prove useful towards development of an efficient photosensitizer for photodynamic therapy [36]. The following polarization properties were experimentally measured to characterize $\mathrm{ZnO}$ NRs to be suitable used as photosensitizer in PDT.

\section{Polarized light transmittance of $\mathrm{ZnO}$ nanorods}

The transmittance behavior of radiations having wavelength in the range of $400-800 \mathrm{~nm}$ indicates that $\mathrm{ZnO}$ nanorods absorb radiations of longer wavelength (red light) and emit the radiations of shorter wavelength (blue light). Rayleigh scattering is dominant for the particles smaller than an excitation wavelength of light 400 .

The decreasing trend of transmission at a wavelength range of $400 \mathrm{~nm}$ to $530 \mathrm{~nm}$ provide Rayleigh scattering in this region and $\mathrm{ZnO}$ nanorods of relatively smaller diameter are present as shown in Figure 2. It is shown that there is slight increase in transmission of light with increase in wavelength from $530 \mathrm{~nm}$ to $800 \mathrm{~nm}$ showing Mie scattering of light is high in this wavelength range and $\mathrm{ZnO}$ nanorods of larger size are randomly distributed.

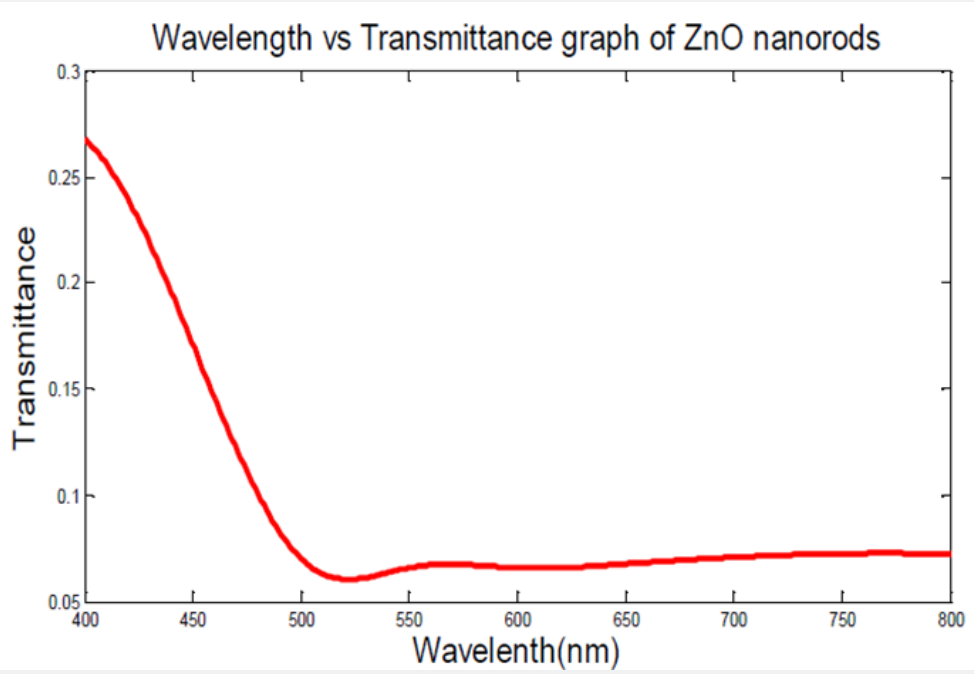

Figure 2: The $\mathrm{ZnO}$ Nanorods transmittance plot at the wavelength of $400-800 \mathrm{~nm}$ of incident light. The Mueller matrix polarized light transmission indicates the optical characterization of the structure of the synthesized material.

\section{Polarized Light Depolarization Index of $\mathrm{ZnO}$ Nanorods}

The depolarization of light provides the mixing of a polarized light coupled into unpolarized light. The depolarization exists only when the incident light is polarized and the degree of polarization of emission light is less than excitation. The surface roughness and bulk scattering value of depolarization index lies in the range from 0 to 1 , corresponds to the unpolarized light compared to polarized light.

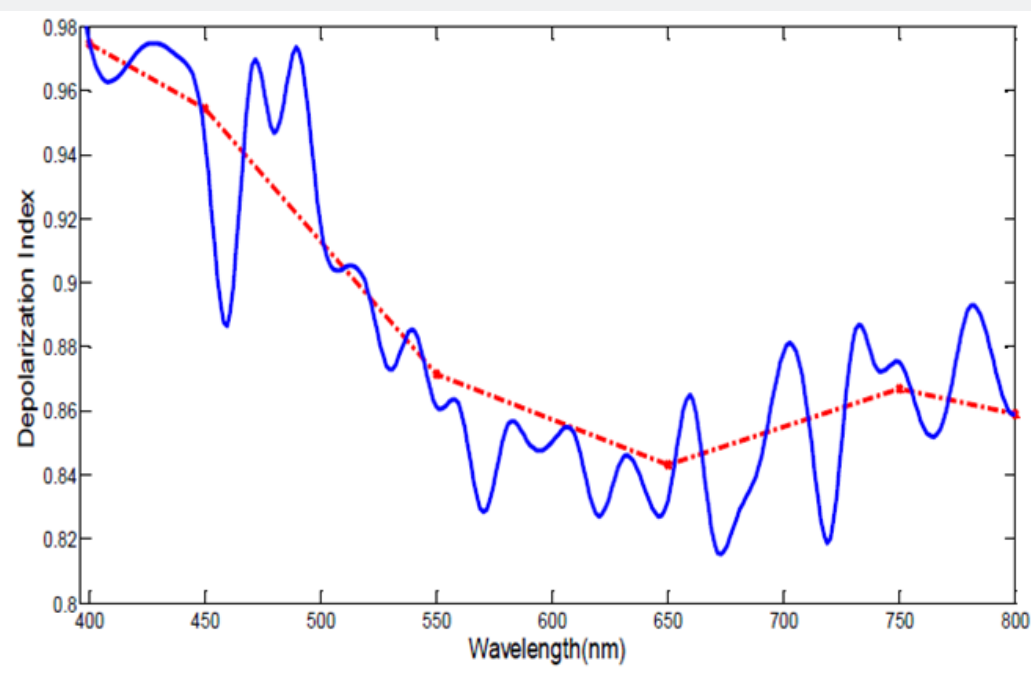

Figure 3: The depolarization index plot of $\mathrm{ZnO}$ nanorods. The Mueller matrix polarized light depolarization with wavelength of light indicates the optical characterization of the structure of the synthesized material. 
The blue line in Figure 3 shows depolarization of light for actual trend obtained and red line represents the average trend. When the wavelength increases from $400 \mathrm{~nm}$ to $650 \mathrm{~nm}$, depolarization index decreases from 0.98 to 0.84 indicating that depolarization is occurring due to bulk scattering of light from randomly distributed $\mathrm{ZnO}$ nanorods. This scattering is Rayleigh scattering pointing to the smaller size of nanorods. There is a slight increase in depolarization index value from 0.84 to 0.86 for light wavelength of $650 \mathrm{~nm}$ to $800 \mathrm{~nm}$. The trend represent that scattering is saturated at $650 \mathrm{~nm}$ wavelength of light.

\section{Polarized Light Retardance Magnitude of ZnO Nanorods}

The polarization retarder introduces a polarization dependent phase change of light between its eigen polarizations in term of retardance. One eigen polarizations shows the maximum transmitted phase and the other shows the minimum transmitted phase, in between these two remaining polarization states show intermediate phase change.

The retardance of $\mathrm{ZnO}$ nanorods is represented in Figure 4, actual results and average of these results represented by blue and red line respectively. Retardance decreases with the increase in wavelength from $445 \mathrm{~nm}$ to $550 \mathrm{~nm}$ and after that, there is a slight change in retardance with the increase in wavelength up to $800 \mathrm{~nm}$. This trend represents light having low frequency and high wavelength passes readily than the higher frequency light providing $\mathrm{ZnO}$ nanorods, strong, compact and composite material.

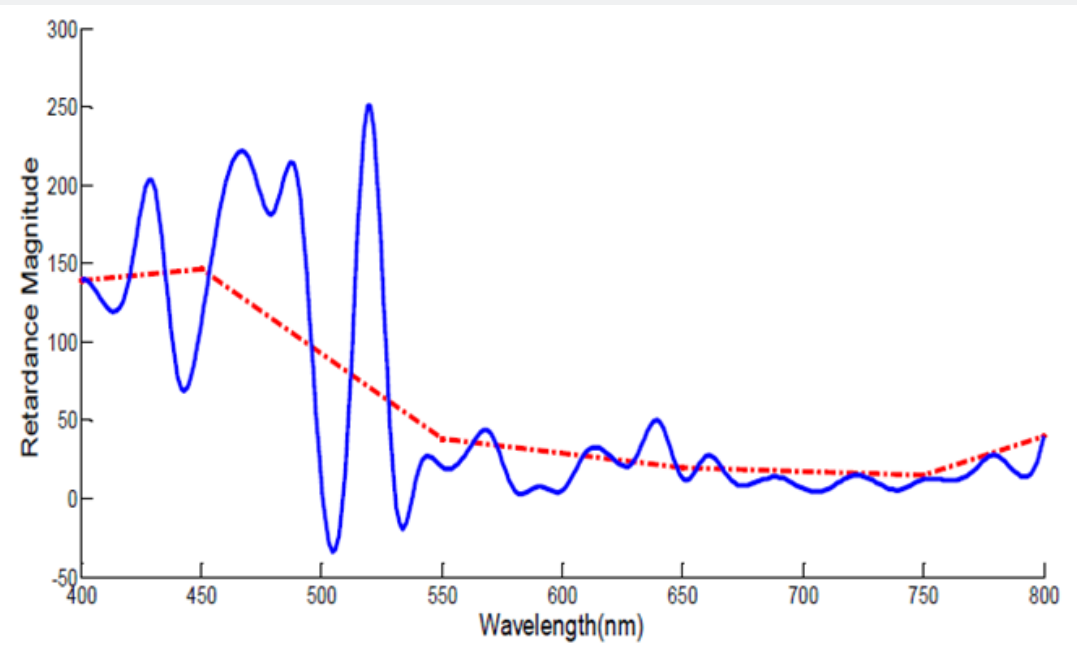

Figure 4: The retardance magnitude plot of $\mathrm{ZnO}$ nanorods. The Mueller matrix polarized light retardance with wavelength of incident light indicates the optical characterization of the structure of the synthesized material.

Polarized Light Diattenuation Magnitude of $\mathrm{ZnO}$ Nanorods

The diattenuation arises when the intensity transmission of the exiting beam is a function of the polarization state of the incident beam. For incident state intensity, transmittance is maximum and minimum for orthogonal state. In Figure 5, we observed that diattenuation magnitude increases slightly with the increase in wavelength from $400 \mathrm{~nm}$ to $750 \mathrm{~nm}$ and changes abruptly at a wavelength range from $750 \mathrm{~nm}$ to $800 \mathrm{~nm}$. This irregular trend indicates that $\mathrm{ZnO}$ nanorods are birefrigant and anistropic 400 .

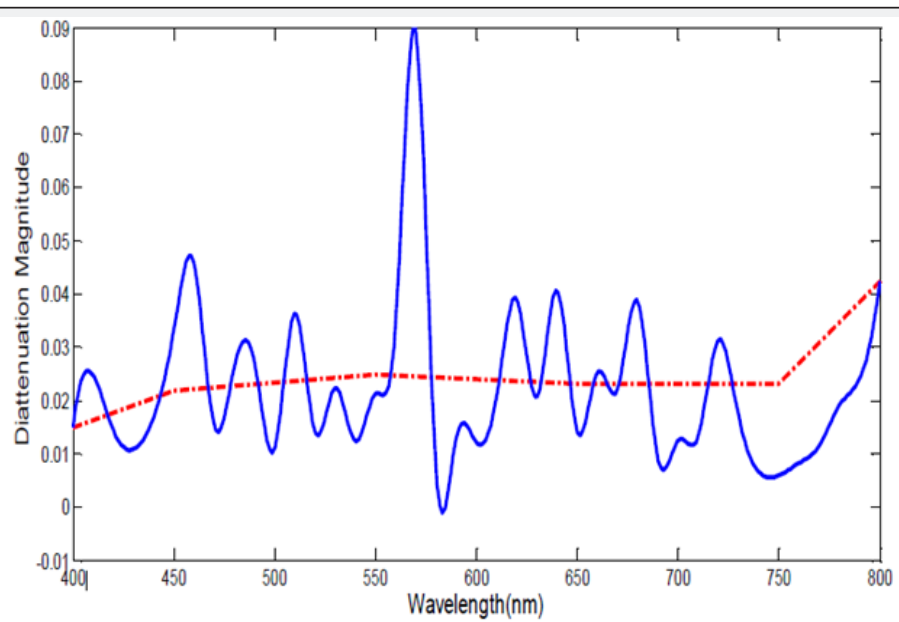

Figure 5: The diattenuation magnitude plot of $\mathrm{ZnO}$ nanorods. The Mueller matrix polarized light Diattenuation with incident light wavelength indicate the optical imaging of the structure of the synthesized material. 


\section{Cancer Therapy \& Oncology International Journal}

\section{Polarized Light Polarizance Magnitude of ZnO Nanorods}

The polarizence is the degree of polarization of the exiting beam when the unpolarized light is incident. Polarizance is just reverse of the depolarization during which unpolrized light transformed into the polarized light. It can be seen in Figure 6, that there is a slight increase in polarizance with the increase in wavelength from $400 \mathrm{~nm}$ to $800 \mathrm{~nm}$ which indicates that ZnO-NRs does not transform the incident unpolarized light to the polarized light due to bulk scattering with the small size particles.

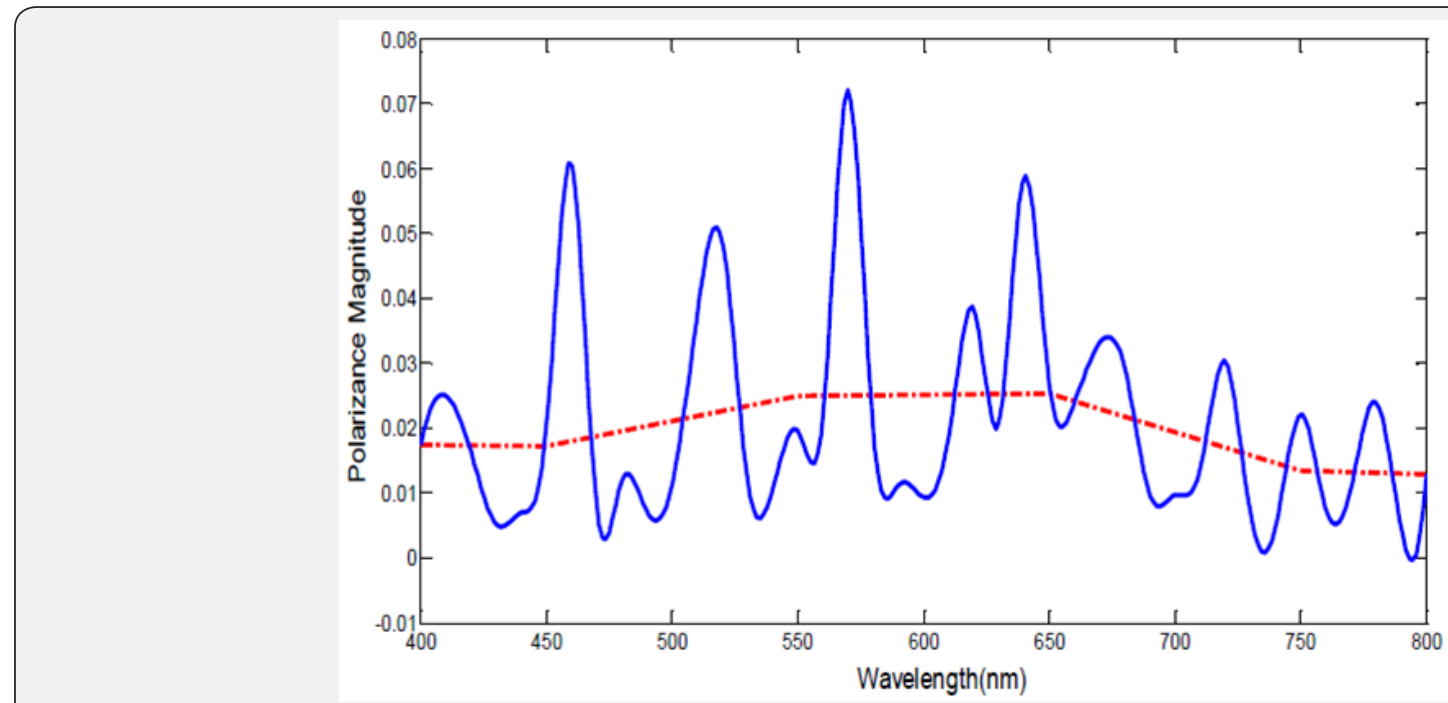

Figure 6: The polarizance magnitude plot of $\mathrm{ZnO}$ nanorods. The Mueller matrix polarized light polarizence with respect to incident light wavelength indicates the optical characterization of the structure of the synthesized material.

Polarized Light Intensity Transmittance model of ZnO Nanorods

The optical properties of $\mathrm{ZnO}$ nanorods directly investigated by intensity transmittance model with 64 elements of polarization measurements at various wavelengths of light as represented in Figure 7. This model reveals information about the diattenuation matrix, retardance matrix, and depolarization matrix. First element of Muller matrix represents direct intensity transmittance. Transmission intensity determined by the first row of the Muller matrix. If diagonal elements of the matrix have same value (intensity), then it represents the retardance Muller matrix and diagonal elements of the depolarization matrix. In this way we have easily determine optical properties by interpreting the Muller matrix elements representing in this model.

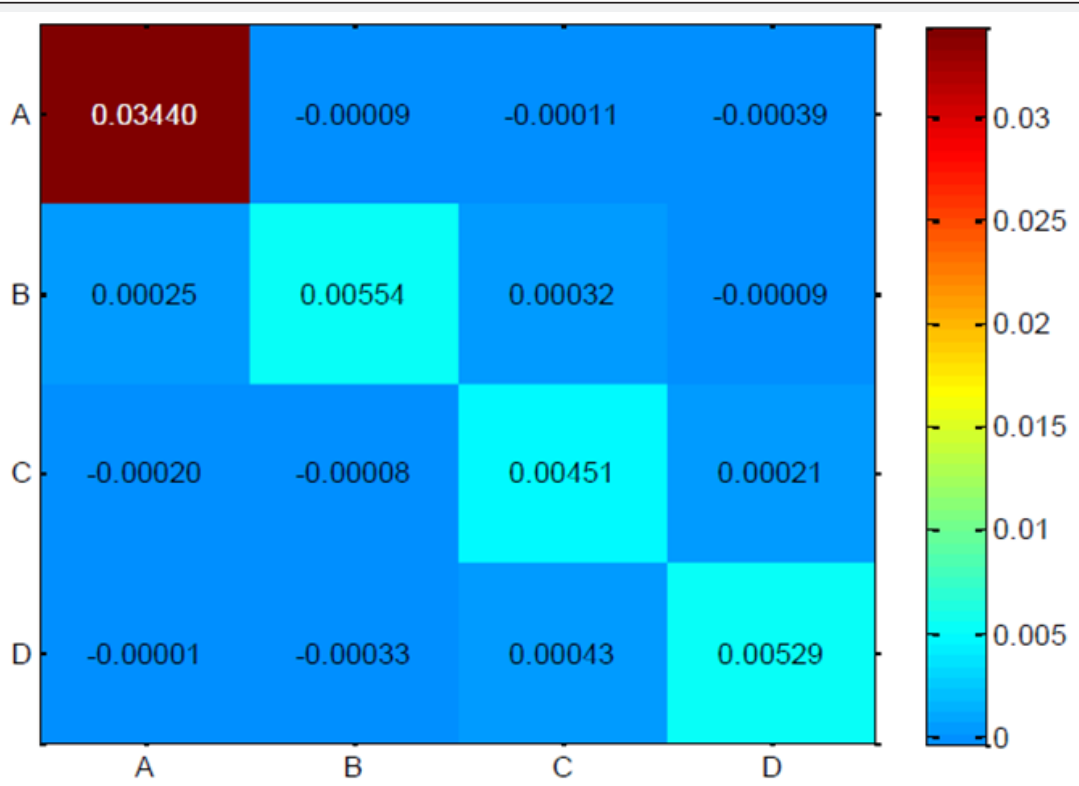

Figure 7: The intensity transmittance Mueller matrix model for $\mathrm{ZnO}$ NRs at $400-800 \mathrm{~nm}$ wavelength. The Mueller matrix polarized light model indicates the optical characterization of the structure of the synthesized material. 


\section{Cancer Therapy \& Oncology International Journal}

The considerably low magnitudes of the diagonal elements imply overall strong depolarizing nature of the scattered light. Non-zero intensities of the elements in the first row and the first column on the other hand, is a manifestation of the linear diattenuation effect. Strong signature of the linear retardance effect is also evident from significant intensities of the off-diagonal elements. In our previous study, Fakhar et al. [6] introduced the concept of Drug delivery into the malignant cell for effectiveness of photosensitizing systems for photodynamic therapy. Apoptotic effect of tumoricidal drugs (ALA and Photofrin $囚$ ) is studied in HepG2 (Hepatucellular carcinoma) cells in the presence and absence of $\mathrm{ZnO}$ NPs using confocal microscopy as well as Neutral Red Assay (NRA). In dark, ZnO NPs conjugated with ALA or Photofrin ${ }^{\circledR}$ has been found to have a remarkable fluorescence in Hepatucellular carcinoma (HepG2) cells [37]. This fact illustrates the great potential of ZnO NPs as biomarker in relevant clinical and biomedical applications.

In another study by Ravindra K Pandey et. al. [38] covers many aspects of PDT and nanotechnology. In his research, he developed multifunctional theranostic nanoparticles, which can carry out both tumor imaging using PET, MRI, and fluorescence and at the same time can mediate tumor therapy via PDT. He also develops models for photosynthetic reaction centers and deeply explores the chemistry of porphyrin-based compounds related to chlorines and bacteriochlorin. Most of the photosensitizers and tumor-imaging agents developed in his group are derived from naturally occurring chlorophyll-a, bacteriochlorophyll a, and several more are in various stages of preclinical and toxicological studies $[37,38]$. The Paras N Parsad et al. [39] presented his research for the preparation, processing, and theoretical modeling of photonic materials for PDT and interested in investigating multifunctional nanostructured materials that can do both therapy and fluorescence imaging and in particular in multiphoton processes and their applications to PDT. Resent results are consistent to our previous findings and other data available in literature.

\section{Conclusion}

The ZnO nanoparticles characterized and used as a photosensitizer along with ALA and Photofrin in HepG2 cells as described in our previous research. The Mueller matrix polarimetric model successfully presented the optical characterization of $\mathrm{ZnO}$ nanorods in term of polarization, depolarization index, retardance, diattanuatioin and polarizence may be helpful for structural changes and particles nature study of $\mathrm{ZnO}$ nanorods. The experimental results conclude that the $\mathrm{ZnO}$ nanorods are strong, highly compatible and dense scattering material suitable candidate for PDT and as a kind of low-cost, low toxic and versatile material, have shown to have a promising future in biological applications. However, there are still challenges in front of researchers to improve or change $\mathrm{ZnO}$ fluorescence, $\mathrm{ZnO}$ bio imaging on the multiple target cells and live animals. Antibacterial and anticancer performances of $\mathrm{ZnO}$ NRs have shown a bright future, but they all require plenty of work before any practical applications.

\section{References}

1. Chatterjee DK, Fong LS, Zhang Y (2008) Nanoparticles in photodynamic therapy: an emerging paradigm. Adv Drug Deliv Rev 60(15): 16271637.

2. Moghimi SM, Hunter AC, Murray JC (2005) Nanomedicine: current status and future prospects. FASEB J 19(3): 311-330.

3. Bechet D, Couleaud P, Frochot C, Viriot ML, Guillemin F, et al. (2008) Nanoparticles as vehicles for delivery of photodynamic therapy agents. Trends Biotechnol 26(11): 612-621.

4. Rasmussen JW, Martinez E, Louka P, Wingett DG (2010) Zinc oxide nanoparticles for selective destruction of tumor cells and potential for drug delivery applications. Expert Opin Drug Deliv 7(9): 1063-1077.

5. Akhtar MJ, Ahamed M, Kumar S, Khan MM, Ahmad J, et al. (2012) Zinc oxide nanoparticles selectively induce apoptosis in human cancer cells through reactive oxygen species. Int J Nanomedicine 7: 845-857.

6. Fakhar e Alam M, Firdous S, Atif M, Khan Y, Zaidi SSZ, et al. (2011) The Potential Applications of ZnO Nanoparticles Conjugated with ALA and Photofrin as a Biomarker in HepG2 Cells. Laser Physics 21(12): 21562164.

7. Atif M, Firdous S, Nawaz M (2010) Laser induced effects in different biological samples, Lasers in Medical Sciences 25(4): 545-550.

8. Khursid M Atif, S Firdous, S Zaidi, R Suleman, M Ikram (2010) Study of the Efficacy of 5 ALA_Mediated Photodynamic Therapy of human Larynx Squamous cell Carcinoma (Hep2c) using 5-aminolevulanic acid in vitro, Laser Physics 20(7): 1673-1678.

9. Kachynski AV, AN Kuzmin, M Nyk, I Roy, PN Prasad (2008) Zinc oxide nanocrystals for nonresonant nonlinear optical microscopy in biology and medicine. J Phys Chem C 112(29): 10721-10724.

10. Hong H, J Shi, Y Yang, Y Zhang, JW Engle, et al. (2011) Cancer-targeted optical imaging with fluorescent zinc oxide nanowires. Nano Letters 11(9): 3744-3750.

11. Hanley C, J Layne, A Punnoose, KM Reddy, I Coombs, et al. (2008) Preferential killing of cancer cells and activated human T cells using ZnO nanoparticles. Nanotechnology 19(29): 5103-5113.

12. Shamaraz Firdous, Shahzad Anwar (2016) Noninvasive optical diagnostic of breast cancer using depolarization of ligh, Optik 127(5): 3035-3038.

13. Shahzad Anwar, Shamaraz Firdous (2016) Optical Diagnosis of Dengue Virus Infected Human Blood using Mueller Matrix Polarimetry. Optics and Spectroscopy 121(2): 322-325.

14. Kamal A, Bashir V, Firdous S, Yasin T, Tariq M, et al. (2016) Optical properties of ultra-high molecular weight polyethylene (UHMWPE): A material of choice for total joint applications. Radiation Physics and Chemistry 118: 102-106.

15. Anwar S, Firdous S, Rehman A, Nawaz M (2015) Raman Optical Diagnostic of Breast Cancer using Raman, Polarimetric Fluorescence Spectroscopy, Laser physics Letter 12(4): 045601.

16. Anwar S, Mubarik M, Waheed A, Firdous S (2015) Polarization Imaging and Characterization of Chitosan for applications in Tissue Engineering. Optik - International Journal for Light and Electron Optics 126: $871-876$

17. Rehman A, Ahmad I, Rehman K, Anwar S, Firdous S, et al. (2015) Optical properties measurement of highly diffusive tissue phantoms for biomedical applications. Laser Physics 25(2): 025605. 


\section{Cancer Therapy \& Oncology International Journal}

18. Aziz T, Firdous S, Rehman T, Khan MA (2013) Polarimetric study of leukemia human peripheral blood smears in $400-800 \mathrm{~nm}$ spectral range, Optik. International Journal for Light and Electron Optics 124(17): 2936-2942.

19. Ahmed M, Ali S, Mehmood S, Khurshid A, Firdous S, et al. (2013) Exvivo assessment of carbon Tetrachloride (CCL4) induced chronic injury using polarized light spectroscopy. Applied Spectroscopy 67(12): 1382-1389.

20. Firdous S, Waqas W, Idrees M, Nawaz M, Ikram M (2013) Polarization Sensitive Optical Imaging of Biomaterials using Mueller matrix Polarimetric Algorithm. Lasers in Engineering 26(3/4): 157-180.

21. Firdous S, Ahmed A, Nawaz M, Ikram M (2013) Optical Characterization of Chitosan for application as an engineered biomaterial, Optik. International Journal for Light and Electron Optics 124(12): 12971302 .

22. Rehman A, Firdous S, Nawaz M, Ahmad M (2012) Optical Parameters Measurement for Diagnostic and Photodynamic Therapy of Human Cervical Adenocarcinoma (HeLa) Cell line. Laser Physics 22(1): 322 326

23. Khurshid A, Firdous S, Ahmat L, Ferraria J, Vollet-Filho JD, et al. (2012) In vivo study of laser irradiation of fractionated drug administration based mechanism for effective photodynamic therapy in rat liver, Laser Physics 22(1): 317-321.

24. Fakhar-e-Alam M, Atif M, Rehman T, Sadia H, Firdous S, et al. (2011) The role of sensitivity of ALA (PpIX)-based PDT on human embryonic kidney cell line (HEK293T). Laser Physics 21(8): 1428-1437.

25. Ozgur U, Alivov YI, Liu C, Teke A, Reshchikov MA, et al. (2005) A comprehensive review of $\mathrm{ZnO}$ materials and devices. Journal of Applied Physics 98(4): 103-108.

26. Gyu-Chul Y, Chunrui W, Won Il P (2005) ZnO nanorods: synthesis, characterization and applications. Semicond Sci Technol 20: S22.

27. Prasad AS (2008) Zinc in human health: Effect of zinc on immune cells. Molecular Medicine 14(5-6): 353-357.

28. Wang ZL (2004) Zinc oxide nanostructures: growth, properties and applications. J Phys Condens Matter 16(25): R829-R858.

29. Firdous S, Hassan K, Ikram M (2005) Formulation of Mueller Matrix and Modeling of depolarizing and scattering of nitrobenzene in a Kerr Cell. Appl Opt 44(7): 1171-1177.
30. Atif M, Firdous S, Khurshid A, Noreen L, Zaidi S, et al. (2009) In vitro study of 5 Aminolevulinic acid (5-ALA) based photodynamic Therapy for apoptosis in human cervical HeLa cell line, Laser Physics Letters 6(12): 886-891.

31. Firdous S, Atif M, Nawaz M (2011) Stokes Vector Determination of Polarized Light Propagation in Turbid Medium. Optics and Spectroscopy 110(3): 438-441.

32. Xiangyang M, Hui Z, Yujie J, Jin X, Deren Y (2005) Sequential occurrence of $\mathrm{ZnO}$ nanopaticles, nanorods, and nanotips during hydrothermal process in a dilute aqueous solution, Materials Letters 59(27): 33933395.

33. Yuzhen L, Lin G, Huibin X, Lu D, Chunlei Y, et al. (2006) Low temperature synthesis and optical properties of small-diameter ZnO Nanorods. J Appl Phys 99: 114302.

34. Firdous S, Fuzail M, Atif M, Nawaz M (2011) Polarimetric Characterization of Ultra-High Molecular Weight Polyethylene (UHMWPE) for Bone Substitute Biomaterials. Optik 122(2): 99-104.

35. Ahsanulhaq Q Umar A, Hahn YB (2007) Growth of aligned ZnO nanorods and nanopencils on $\mathrm{ZnO} / \mathrm{Si}$ in aqueous solution: growth mechanism and structural and optical properties. Nanotechnology 18(11): 115603.

36. Khan Y, Mehmood M, Ahmad M, Riaz Khan J, Firdous S (2010) Low temperature synthesis of fluorescent $\mathrm{ZnO}$ nanoparticles. Applied Surface Science 257: 1756 -1761.

37. Wang S, Fan W, Kim G, Hah HJ, Lee YE, et al. (2011) Novel methods to incorporate photosensitizers into nanocarriers for cancer treatment by photodynamic therapy. Lasers Surg Med 43(7): 686-695.

38. Kozyrev AN, Chen Y, Goswami LN, Tabaczynski WA, Pandey RK (2006) Characterization of porphyrins, chlorins, and bacteriochlorins formed via allomerization of bacteriochlorophyll a. Synthesis of highly stable bacteriopurpurinimides and their metal complexes. J Org Chem 71(5): 1949-1960.

39. Ohulchanskyy TY, Roy I, Goswami LN, Chen Y, Bergey EJ, et al. (2007) Organically modified silica nanoparticles with covalently incorporated photosensitizer for photodynamic therapy of cancer. Nano Lett 7(9): 2835-2842.

\section{Your next submission with Juniper Publishers} will reach you the below assets

- Quality Editorial service

- Swift Peer Review

- Reprints availability

- E-prints Service

- Manuscript Podcast for convenient understanding

- Global attainment for your research

- Manuscript accessibility in different formats

( Pdf, E-pub, Full Text, Audio)

- Unceasing customer service

Track the below URL for one-step submission https://juniperpublishers.com/online-submission.php 